Related content

Spectral distributions of the scattered photons within an acceptance angle in Thomson scattering

Wang Jun and Huang Wen-Hui

Preliminary experimental study and simulation of an energy-tunable quasimonochromatic laser-Compton X/-ray

Luo Wen, Xu Wang, Zhuo Hong-Bin et al.

- Coherent x-ray and gamma-ray generation A A Risbud

View the article online for updates and enhancements. 


\title{
Two-colour X-gamma ray inverse Compton back-scattering source
}

\author{
I. Drebot ${ }^{1}$, V. Petrillo ${ }^{1,2}$ and L. Serafini ${ }^{1}$ \\ 1 INFN-Milano - Via Celoria 1620133 Milano, Italy \\ 2 Università degli Studi di Milano - Via Celoria 1620133 Milano, Italy
}

received 14 September 2017; accepted in final form 25 November 2017 published online 28 December 2017

PACS $41.50 .+\mathrm{h}-\mathrm{X}$-ray beams and x-ray optics

PACS 41.60.Ap - Synchrotron radiation

PACS $41.60 .-\mathrm{m}$ - Radiation by moving charges

\begin{abstract}
We present a simple and new scheme for producing two-colour Thomson/Compton radiation with the possibility of controlling separately the polarization of the two different colours, based on the interaction of one single electron beam with two light pulses that can come from the same laser setup or from two different lasers and that collide with the electrons at different angle. One of the most interesting cases for medical applications is to provide two X-ray pulses across the iodine $K$-edge at $33.2 \mathrm{keV}$. The iodine is used as contrast medium in various imaging techniques and the availability of two spectral lines accross the $K$-edge allows one to produce subtraction images with a great increase in accuracy.
\end{abstract}

Copyright (C) EPLA, 2017

The development of X-ray sources characterized by high versatility, large spectral flux and tunability, opens the way for a real breakthrough in a wide number of scientific and technical fields. One of the most promising formats in which radiation can be delivered to users consists in wave packets containing two different spectral lines with adjustable frequencies and time separation. Pairs of coloured X-ray pulses allow one to deepen the fundamental understanding of the properties of materials and living systems, by probing the matter on atomic scale in space and time [1] or performing pump and probe experiments of structural dynamics, a class of experiments designed to monitor the ultrafast changes in atomic, electronic and magnetic structures [2-4]. Processes as chemical reactions or excitations and structural transformations in matter are excited by a first pulse and then probed with a second one of another colour. The time scale for such dynamics can range from $10 \mathrm{fs}$ in ultrafast processes as the dissociative ionization [5], to hundreds of femtoseconds for less energetic chemical mechanisms [6,7]. Another important issue is the future colour X-ray technology. Colour X-ray imaging will provide significant development to screening or diagnostic radiography, because the colour components contain extra information and allow to discriminate the chemical composition of the absorbing tissues [8]. Experiments on dual colour have been recently carried on with free-electron lasers (FELs) as radiation sources $[4,9-11]$ and promising proposals aimed to generate two-colour X-ray emission in Compton sources $[12,13]$ have been investigated. Thomson and Compton sources, even though less brilliant than FELs, produce radiation with short wavelength, high power, ultrashort time duration, large transverse coherence and tunability, full polarization control, ensuring limited costs of construction and maintenance and dimensions compatible with the space that can be allocated in hospitals and medical centres. Existing Thomson sources [14-21] are important tools for generating tunable quasimonochromatic $\mathrm{X} /$ gamma rays suitable for applications in crystallography, plasma, high energy, matter physics and nuclear photonics and in the advanced biomedical imaging as demonstrated by the wide number of experiments on phase contrast imaging [18,22], microtomography [22], $K$-edge techniques $[13,23]$ on biological and human samples. In this paper we present a simple and new scheme for producing two-colour Thomson/Compton radiation with the possibility of controlling independently the polarization of the two beamlets. It is based on the interaction of one single electron beam with two light pulses that can come from the same laser setup or from two different lasers colliding with the electrons at different angle. One of the most interesting cases for medical applications is to provide two $\mathrm{X}$-ray pulses across the iodine $K$-edge at $33.2 \mathrm{keV}$. The iodine is used as contrast medium in various imaging techniques and the availability of two spectral lines below and beyond the $K$-edge allows to produce subtraction images 


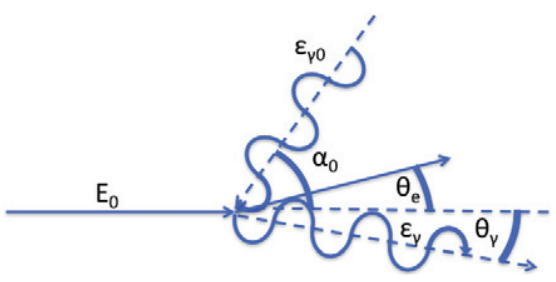

Fig. 1: (Colour online) Kinematic of the Compton back scattering.

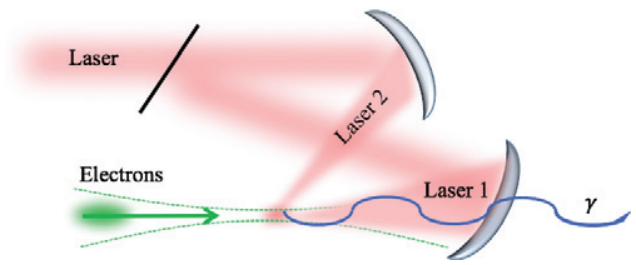

Fig. 2: (Colour online) Scheme of the use of a split laser sent to the interaction point at two different interaction angle.



Fig. 3: (Colour online) Dependence of the maximal energy of the scattered photons from the initial scattering angle $\alpha_{0}$ for $\gamma=84, \varepsilon_{\gamma 0}=1.2 \mathrm{eV}$.

with a great increase in accuracy. The application to this range of X-rays is presented and discussed.

The Thomson or inverse Compton scattering is the process occurring when an electron belonging to a highbrightness electron beam collides with the photons of a laser pulse, generating $\mathrm{X}$ or gamma radiation. The geometry of the scattering is represented in fig. 1 , where $\alpha_{0}$ is the interaction angle of the scattering.

The radiation energy is upshifted with respect to the lasers's one by the relation

$$
\varepsilon_{\gamma m}=\frac{4 \gamma^{2} \varepsilon_{L} \cos ^{2} \frac{\alpha_{0}}{2}}{4 \gamma \frac{\varepsilon_{L}}{m c^{2}} \cos ^{2} \frac{\alpha_{0}}{2}+1} \approx 4 \gamma^{2} \varepsilon_{L} \cos ^{2} \frac{\alpha_{0}}{2},
$$

where $\varepsilon_{L}$ is the laser photon energy, $\gamma$ the electron Lorentz factor and $\varepsilon_{\gamma}$ the emitted photon energy and the electron recoil term can be disregarded. The scheme we are proposing for producing two-colour radiation is based on the interaction of the electron beam with two light pulses that can come from the same laser setup or from two different lasers and that collide with the electrons at different
Table 1: Parameters of electron beam and laser system.

\begin{tabular}{|c|c|}
\hline \multicolumn{2}{|l|}{ Electron beam parameters } \\
\hline Electrons mean energy (Mev) & 43.2 \\
\hline Bunch charge (nC) & 1 \\
\hline Bunch length rms $(\mu \mathrm{m})$ & $10^{3}$ \\
\hline Nominal normalized $\epsilon_{n x}, \epsilon_{n y}(\mathrm{~mm} \cdot \mathrm{mrad})$ & $0.99,0.98$ \\
\hline Nominal relative energy spread $\sigma_{e}(\%)$ & 0.5 \\
\hline Focal spot size $\sigma_{x}, \sigma_{y}(\mu \mathrm{m})$ & 15,15 \\
\hline \multicolumn{2}{|l|}{ Laser parameters 1} \\
\hline Laser pulse energy $(\mathrm{J})$ & 0.15 \\
\hline Laser pulse length (ps) & 1 \\
\hline Laser focal spot size w0 RMS $(\mu \mathrm{m})$ & 60 \\
\hline Collision angle (deg) & 0 \\
\hline STOKES parameters & $(0,0,-1)$ \\
\hline \multicolumn{2}{|l|}{ Laser parameters 2} \\
\hline Laser pulse energy $(\mathrm{J})$ & 0.15 \\
\hline Laser pulse length (ps) & 1 \\
\hline Laser focal spot size w0 RMS $(\mu \mathrm{m})$ & 24 \\
\hline Collision angle (deg) & 30 \\
\hline STOKES parameters & $(0,0,+1)$ \\
\hline
\end{tabular}

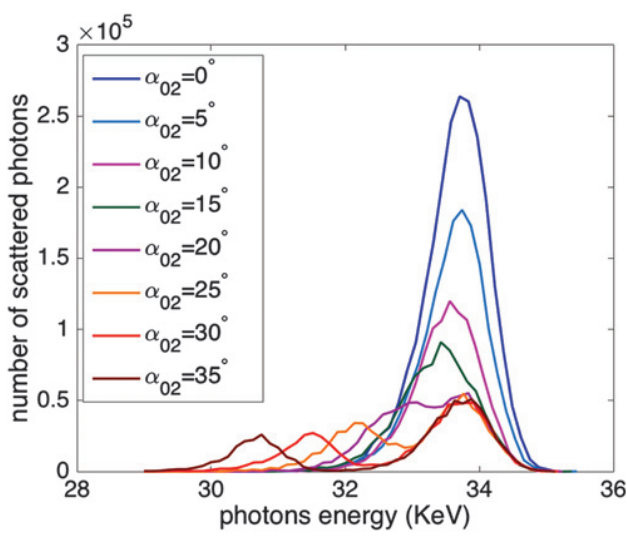

Fig. 4: (Colour online) Spectra of the scattered radiations for different initial angles for the second laser $\alpha_{02}$.

angle, as shown in fig. 2. If the first scattering is headon, the angle of the second one is chosen in order to fix the relative separation between the two radiation pulses $\Delta \varepsilon / \varepsilon=\sin ^{2}\left(\alpha_{0} / 2\right)$. Figure 3 shows the dependence of the scattered photon energy on the angle, for typical values of a Thomson source (see table 1) such as the STAR Project [24].

Figure 4 presents the evaluation of the spectrum of the scattered photons for different values of the angle of the second laser $\alpha_{02}$ collimated into the fixed acceptance angle performed with the Monte Carlo code CAIN [25].

As can be seen, the radiation energy for $\alpha_{0}=0$ is about $E_{1}=34 \mathrm{keV}$, above the iodine $K$-edge. A separation 


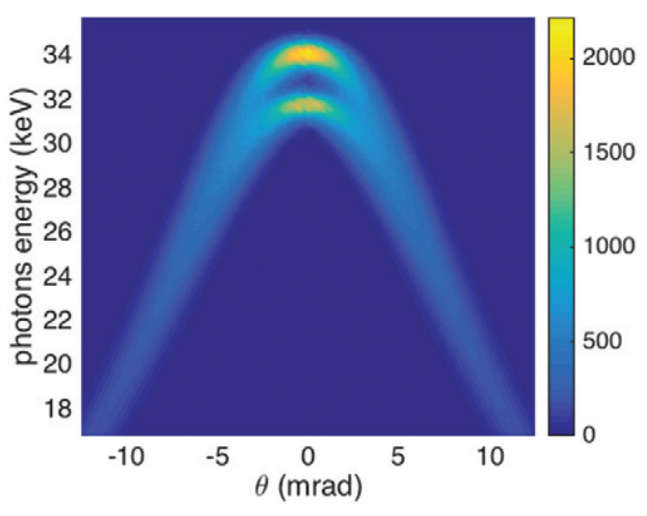

Fig. 5: (Colour online) Energy angular distribution of the scattered radiation for $\alpha_{02}=30 \mathrm{deg}$.

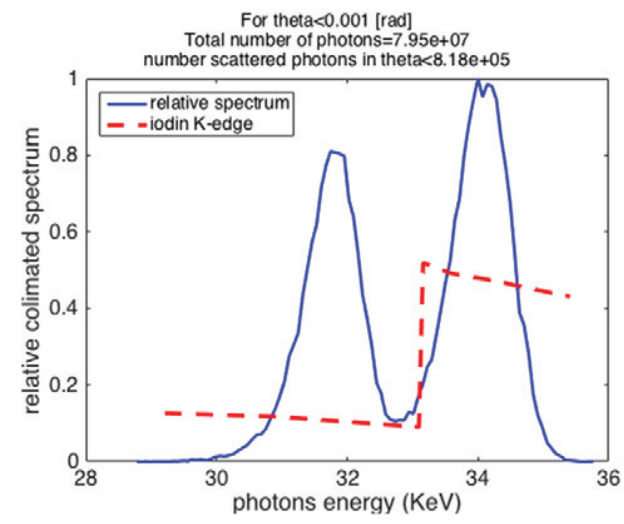

Fig. 6: (Colour online) Relative spectrum of the scattered radiation for $\alpha_{02}=30 \mathrm{deg}$.

$\Delta \varepsilon / \varepsilon \approx 6.5 \%$ between the energies of the two pulses means, for instance, to operate with one head-on collision and the other one at about $30^{\circ}$. In this case, the second line will be at $E_{2}=32 \mathrm{keV}$. Furthermore, the number of scattered photons $N$ in a Thomson/Compton scattering at a generic angle $\alpha_{0}$ collimated in an acceptance angle $\Psi=\gamma \theta_{\max }$ can be obtained on the basis of the luminosity as

$N^{\Psi}=$

$\frac{f N_{e} N_{L} \int^{\Psi} \mathrm{d} \Psi^{\prime} \frac{\mathrm{d} \sigma}{\mathrm{d} \Psi^{\prime}}}{2 \pi \sqrt{\sigma_{y, e}^{2}+\sigma_{y, L}^{2}} \sqrt{\sigma_{x, e}^{2}+\sigma_{x, L}^{2}+\left(\sigma_{z, e}^{2}+\sigma_{z, L}^{2}\right) \tan ^{2}\left(\frac{\alpha_{0}}{2}\right)}}$,

where $\int^{\Psi} \mathrm{d} \Psi^{\prime} \frac{\mathrm{d} \sigma}{\mathrm{d} \Psi^{\prime}}$ is the Compton cross-section as a function of the acceptance angle $\Psi[26], N_{e}, N_{L}$ are the number of interacting electrons and laser photons, $\sigma_{x}\left(\sigma_{x, L}\right)$ and $\sigma_{y}\left(\sigma_{y, L}\right)$ are the rms electron (laser) transverse dimensions at waist, $\sigma_{z}\left(\sigma_{z, L}\right)$ is the electron (laser) beam length and $\theta_{\max }$ is the maximum acceptance angle. If the two radiation pulses are required to have comparable photon numbers, the lengths $\sigma_{z}\left(\sigma_{z, L}\right)$ should be as short as possible and the two laser pulses should be focused in a different way, in particular the first laser beam was focused

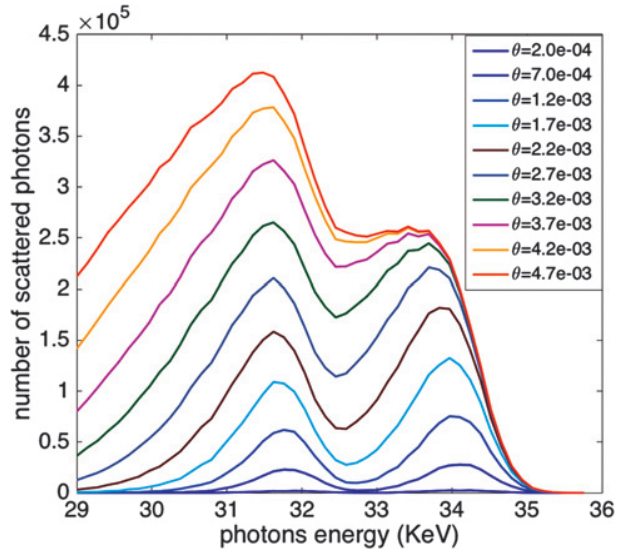

Fig. 7: (Colour online) Spectra of the scattered radiations for different collimation angles $\theta$.

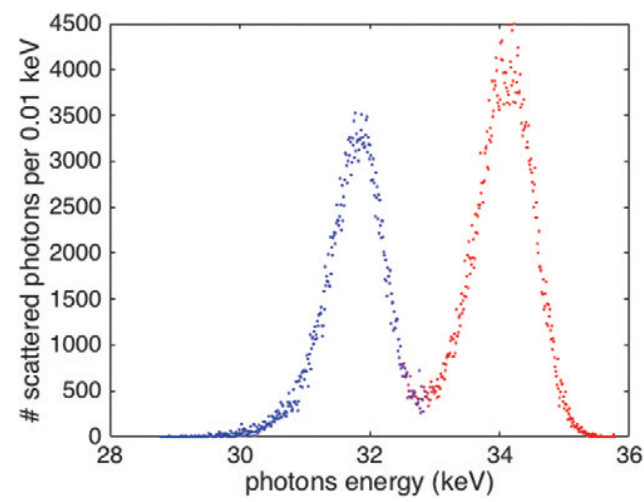

Fig. 8: (Colour online) Spectra of the scattered radiations for different collimation angles $\theta$. Colours represent different polarisations.

at $w 0_{L 1}=60 \mu \mathrm{m}$ and the other one at $w 0_{L 2}=24 \mu \mathrm{m}$ as shown in table 1 . In fig. 5 the total photon phase space is reported, for $\alpha_{1}=0$ and $a_{2}=30^{\circ}$. Figure 6 presents the spectrum of the radiation collimated within an acceptance angle $\theta=1 \mathrm{mrad}$, with a good balance between the two spectral peaks. Another quantity that has to be controlled for separating the two spectral lines is the acceptance angle. Figure 7 presents the spectrum of scattered radiations as a function $\theta$ and shows that only for acceptance angles lower than $\left(E_{2}-E_{1}\right) / E$ the two spectral lines present a reasonable separation.

For producing two radiation pulses of different polarisation $[27,28]$ we can propose to insert a polarization rotator along the trajectory of one of the lasers, thus providing the possibility to get two colours also with different polarisations. In fig. 8 we present the spectrum of the scattered photons from two lasers with different polarisations.

In this work we present a new scheme to produce twocolour X-rays based on compact Compton sources. This scheme consists in the use of two laser pulses impinging on the same electron beam at two different angles, with frequencies given by formula (1). The potentialities of scattered radiations can be improved by using a different 
polarisation of the initial laser pulses. This scheme can be extended to the production of a sequence of two X-ray pulses with different colours separated also in time. In fact, it is possible to change and adjust at will the temporal delay between two laser pulses, which, in turn, equals the time separation between two successive electron bunches. This is of paramount importance for adjusting the time needed by the detectors to record and load the two images at two different colours, which is mandatory for digital subtraction This technique is thought for devices based on electron-laser scattering in free space, with optimized single-shot parameters and relatively low repetition rate, such as for instance the source STAR. The application in cavity, in fact, would be much more complicated to implement and deserves further analysis. The two-colour imaging by Monte Carlo technique simulation through a phantom will be analysed in future works.

\section{REFERENCES}

[1] Gunther C. M., Pfau B., Mitzner R., Siemer B., Roling S., Zacharias H., Kutz O., Rudolph I., Schondelmaier D., Treusch R. and Eisebitt S., Nat. Photon., 5 (2011) 99.

[2] Tavella F., Stojanovic N., Geloni G. and Gensch M., Nat. Photon., 5 (2011) 162.

[3] Ding Y., Decker F.-J., Emma P., Feng C., Field C., Frisch J., Huang Z., Krzywinski J., Loos H., Welch J., Wu J. and Zhou F., Phys. Rev. Lett., 109 (2012) 254802.

[4] Allaria E., Bencivenga F., Borghes R., Capotondi F., Castronovo D., Charalambous P., Cinquegrana P., Danailov M. B., De Ninno G., Demidovich A., Di Mitri S., Diviacco B., Fausti D., Fawley W. M., Ferrari E., Froehlich L., Gauthier D., Gessini A., Giannessi L., Ivanov R., Kiskinova M., Kurdi G., Mahieu B., Mahne N., Nikolov I., Masciovecchio C., Pedersoli E., Penco G., Raimondi L., Serpico C., Sigalotti P., Spampinati S., Spezzani C., Svetina C., Trovò M. and Zangrando M., Nat. Commun., 4 (2013) 2476.

[5] Bocharova I. A., Alnaser A. S., Thumm U., Niederhausen T., Ray D., Cocke C. L. and Litvinyuk I. V., Phys. Rev. A, 83 (2011) 013417.

[6] Tao H., Allison T. K., Wright T. W., Stooke A. M., Khurmi C., van Tilborg J., Liu Y., Falcone R. W., Belkacem A. and Martinez T. J., J. Chem. Phys., 134 (2011) 244306.

[7] Allison T. K., Tao H., Glover W. J., Wright T. W., Stooke A. M., Khurmi C., van Tilborg J., Liu Y., Falcone R. W., Martínez T. J. and Belkacem A., J. Chem. Phys., 136 (2012) 124317.

[8] Dierickx B., Yao Q., Witvrouwen N., Uwaerts D., Vandewiele S. and Gao P., Sensors, 16 (2016) 764.

[9] Marinelli A., Ratner D., Lutman A. A., Turner J., Welch J., Decker F. J., Loos H., Behrens C., Gilevich S., Miahnahri A. A., Vetter S., Maxwell T. J., Ding Y., Coffee R., Wakatsuki S. and Huang Z., Nat. Commun., 6 (2015) 6369.
[10] Lutman A. A., Coffee R., Ding Y., Huang Z., Krzywinski J., Maxwell T., Messerschmidt M. and Nuhn H.-D., Phys. Rev. Lett., 110 (2013) 134801.

[11] Petrillo V., Anania M. P., Artioli M., Bacci A., Bellaveglia M., Chiadroni E., Cianchi A., Ciocci F., Dattoli G., Di Giovenale D., Di Pirro G., Ferrario M., Gatti G., Giannessi L., Mostacci A., Musumeci P., Petralia A., Pompili R., Quattromini M., Rau J. V., Ronsivalle C., Rossi A. R., Sabia E., Vaccarezza C. and Villa F., Phys. Rev. Lett., 111 (2013) 114802.

[12] Petrillo V., Bacci A., Curatolo C., Ferrario M., Gatti G., Maroli C., Rau J. V., Ronsivalle C., Serafini L., Vaccarezza C. and Venturelli M., Phys. Rev. ST Accel. Beams, 17 (2014) 020706.

[13] Hwang Y., Barty C., Gibson D., Marsh R. and TAJima T., Electron Beam Diagnosis Using K-edge Absorption of Laser-Compton Photons, in Proceedings of the International Particle Accelerator Conference (IPAC'17), Copenhagen, Denmark, 14-19 May, 2017, No. 8 (JACoW, Geneva, Switzerland) 2017, pp. 473-476, https://doi.org/10.18429/JACoW-IPAC2017-MOPAB146, http://jacow.org/ipac2017/papers/mopab146.pdf.

[14] Sakai Y., Gadjev I., Hoang P., Majernik N., Nause A., Fukasawa A., Williams O., Fedurin M., Malone B., Swinson C., Kusche K., Polyanskiy M., Babzien M., Montemagno M., Zhong Z., Siddons P., Pogorelsky I., Yakimenko V., Kumita T., Kamiya Y. and Rosenzweig J. B., Phys. Rev. Accel. Beams, 20 (2017) 060701.

[15] Achterhold K., Bech M., Schleede S., Potdevin G., Ruth R., Loewen R. and Pfeiffer F., Sci. Rep., 3 (2013) 1313.

[16] Eggl E., Mechlem K., Braig E., Kulpe S., Dierolf M., Günther B., Achterhold K., Herzen J., Gleich B., Rummeny E., NoËl P. B., Pfeiffer F. and Muenzel D., Sci. Rep., 7 (2017) 42211.

[17] Akagi T., Kosuge A., Araki S., Hajima R., Honda Y., Miyajima T., Mori M., Nagai R., Nakamura N., Shimada M., Shizuma T., Terunuma N. and Urakawa J., Phys. Rev. Accel. Beams, 19 (2016) 114701.

[18] Kuroda R., Toyokawa H., Yasumoto M., IkeuraSekiguchi H., Koike M., Yamada K., Yanagida T., NAKajyo T., SAKai F. and Mori K., Nucl. Instrum. Methods Phys. Res. Sec. A, 637 (2011) S183.

[19] Brown W. J., Anderson S. G., Barty C. P. J., Betts S. M., Booth R., Crane J. K., Cross R. R., Fittinghoff D. N., Gibson D. J., Hartemann F. V., Hartouni E. P., Kuba J., Le Sage G. P., Slaughter D. R., Tremaine A. M., Wootton A. J., Springer P. T. and Rosenzweig J. B., Phys. Rev. ST Accel. Beams, 7 (2004) 060702.

[20] Vaccarezza C., Alesini D., Anania M. P., Bacci A., Biagioni A., Bisesto F., Bellaveglia M., Cardarelli P., Cardelli F., Cianchi A., Chiadroni E., Croia M., Curcio A., Delogu P., Di Giovenale D., Di Domenico G., Di Pirro G., Drebot I., Ferrario M., Filippi F., Gallo A., Galletti M., Gambaccini M., Giribono A., Golosio B., Li W., Mostacci A., Oliva P., Palmer D., Petrillo V., Petrarca M., Pioli S., Piersanti L., Pompili R., Romeo S., Rossi A. R., Scifo J., Serafini L., Suliman G. and Villa F., 
Nucl. Instrum. Methods Phys. Res. Sect. A, 829 (2016) 237.

[21] Du Y., Yan L., Hua J., Du Q., Zhang Z., Li R., Qian H., Huang W., Chen H. and Tang C., Rev. Sci. Instrum., 84 (2013) 053301.

[22] Bech M., Bunk O., David C., Ruth R., Rifkin J., Loewen R., Feidenhans'L R. and Pfeiffer F., J. Synchrotr. Radiat., 16 (2009) 43.

[23] Golosio B., Endrizzi M., Oliva P., Delogu P., Carpinelli M., Pogorelsky I. and Yakimenko V., Appl. Phys. Lett., 100 (2012) 164104.

[24] BACCi A. et al., The Star Project, in Proceedings of the 5th International Particle Accelerator Conference (IPAC
2014): Dresden, Germany, June 15-20, 2014, 2014, p. WEPRO115, http://jacow.org/IPAC2014/papers/ wepro115.pdf.

[25] Yokoya K., User Manual of CAIN, version 2.40 (2009).

[26] Curatolo C., Drebot I., Petrillo V. and Serafini L., Phys. Rev. Accel. Beams, 20 (2017) 080701.

[27] Petrillo V., Bacci A., Curatolo C., Drebot I., Giribono A., Maroli C., Rossi A. R., Serafini L., Tomassini P., Vaccarezza C. and Variola A., Phys. Rev. ST Accel. Beams, 18 (2015) 110701.

[28] Drebot I., Karnaukhov I. and Zelinsky A., Vopr. At. Nauki Tekh. Yad-fiz. issled., 42 (2004) 137. 